\title{
Fertility Preservation in Females: Emerging Era of Ice Age
}

\author{
Shalini Gainder ${ }^{1}$, Japleen Kaur ${ }^{2}$
}

\begin{abstract}
Fertility preservation in malignancy and certain benign conditions is gaining momentum, with advancement in the techniques for the same. It has become more relevant with improving treatment of cancers and increasing life expectancy. Reproductive potential is an important aspect of a woman's life. It is the responsibility of the care provider to be aware of the options of fertility preservation. The various techniques available for the purpose of fertility preservation in females range from oocyte/embryo/ovarian tissue cryopreservation to fertility-preserving surgeries, and the choice of procedure depends on numerous patient and treatment factors. Healthcare provider must be able to assess eligibility of the patient for preservation of fertility potential, carefully balancing the risk and benefit of the same, in relation to the cancer treatment. There are other ethical and legal concerns, which must be discussed in detail with the woman. Therefore, counseling is an integral part of the process.

Keywords: Cryofreezing, Embryo, Fertility preservation, Gonadotoxic, Non-Hodgkins, Oncofertility, Oocyte.

Journal of Postgraduate Medicine Education and Research (2019): 10.5005/jp-journals-10028-1343
\end{abstract}

\section{INTRODUCTION}

With the recent possibility of preserving female fertility, there has been an increased demand for the same in oncologic and nononcologic diseases where the disease itself or its treatment poses a threat to ovarian reserve or future fertility.

Fertility preservation must be considered as an integral part of counseling when a child or a woman of reproductive age is planned for any kind of therapy that may affect her future fertility aspects especially gonadotoxic chemotherapeutic agents or radiotherapy. There are certain limitations and the inability to provide such kind of treatment, whether due to severity of disease or due to nonavailability, must be recorded to safeguard the interest of the woman and the treating physician.

The type of fertility preservation technique that can be offered varies with the age of the woman, pubertal status, single or availability of male partner, etc. These factors shall determine whether the option of preserving the gametes, the oocytes, or the fertilized embryos is to be employed.

The aim of this review is to elaborate the available options and techniques of fertility preservation in females as per the various indications and patient factors.

\section{Oncofertility}

Oncofertility is a novel branch of medicine, which deals with preserving reproductive potential while balancing life-saving cancer treatment. ${ }^{1}$ Selection of suitable candidates for fertility preservation requires consideration of certain factors like the age of the patient, likelihood of sterility after treatment, and estimated life expectancy. For more details, the Edinburgh criteria can be referenced. ${ }^{2}$ Potential candidates include breast cancer survivors, patients suffering from Hodgkin lymphoma, Ewing sarcoma, acute lymphoblastic leukemia, breast cancer gene (BRCA) carriers, or those treated with chemotherapy or radiotherapy for various other malignancies in young age.

\section{Fertility Preservation in Benign Diseases}

Recent research is now focused to preserve fertility in various benign diseases where the future fertility is decreased either due to treatment or due to hematological or autoimmune diseases like systemic lupus erythematosus, refractory glomerulonephritis, and

\begin{abstract}
1,2 Department of Obstetrics and Gynaecology, Postgraduate Institute of Medical Education and Research, Chandigarh, India

Corresponding Author: Shalini Gainder, Department of Obstetrics and Gynaecology, Postgraduate Institute of Medical Education and Research, Chandigarh, India, Phone: +91 9815555732, e-mail: sgainder@gmail.com

How to cite this article: Gainder S, Kaur J. Fertility Preservation in Females: Emerging Era of Ice Age. J Postgrad Med Edu Res 2019;53(4):179-181.
\end{abstract}

Source of support: Nil

Conflict of interest: None

inflammatory bowel disease. ${ }^{3}$ It is a known fact that autoimmune diseases increase the risk of premature ovarian insufficiency (POI) or failure. Severe endometriosis involving the ovaries may also warrant fertility preservation as it decreases the ovarian reserve and the chances to become infertile are high.

\section{Aging and Fertility Preservation}

The demand to delay the aging clock whether by hormonal supplementation or otherwise has opened new aspects in the field of medicine. The woman is now more oriented toward career building and thereby delaying childbearing. This delay affects their reproductive capacity. It creates fear of losing fertility therefore having an impact on them psychologically. It is now considered ethical to allow fertility preservation in women with such demands and offer them advise and counseling and help them preserve their gametes for future procreation. ${ }^{4,5}$

\section{Various Options for Fertility Preservation \\ Embryo Freezing}

This is the most well-established method of fertility preservation in women who are married or have a partner. It involves stimulation of the ovary with the ovarian stimulating drugs following which the desired number of ovarian follicles are stimulated and retrieved by aspirating the ovary. Then, these oocytes are fertilized in vitro, with 
the male partners sperms, and the fertilized oocytes develop into embryos in a well-controlled environment that is cryofreezed by vitrification (rapid freezing) in liquid nitrogen for future use. This is done as an emergency method of fertility preservation just prior to giving chemotherapy or definitive surgery.

Woman's fitness to undergo ovarian stimulation needs to be ascertained prior to in vitro fertilization (IVF) as it leads to raised estrogen levels and predisposing an already suffering female to added risks and also delay chemotherapy by 15-20 days. Therefore, it is not always suitable to perform an IVF cycle prior to chemotherapy. Ethical and legal dilemmas especially regarding disposal of embryos in the event of death of the woman need to be discussed at the time of decision for IVF. ${ }^{5}$

The luteal-phase stimulation protocols or the random start protocols make it possible to start stimulation of ovaries without delay and embryos are formed from retrieved oocytes and cryofrozen for future use. ${ }^{6}$

\section{Oocyte Freezing}

Oocyte freezing has been possible in recent years and this option can be preferred in women who do not have partners. In 1986, first live birth was achieved from previously frozen oocyte. With improvement in techniques and use of the ultra-rapid freezing technique called vitrification, this method is now no more classified as experimental as previously labeled. ${ }^{5}$ Just prior to exposure to chemotherapy in women with malignancy, ovary can be stimulated with gonadotropins or with aromatase inhibitors where estrogens excess needs to be avoided and oocytes retrieved, and they are further vitrified and stored. Post-thaw retrieval and fertilization have consistently improved over the years. This cannot be applied in prepubertal girls. Oocyte freezing is being performed globally for malignancy and also for benign indications. In fact, there is an ever-increasing demand for oocyte freezing in women who have not chosen their partners and the biological clock of ovarian aging is clicking. This is now ethically permissible and referred to as planned oocyte cryopreservation. It is also being referred as social egg freezing. ${ }^{7,8}$ This is legitimate and provides her autonomy. ${ }^{5}$ However, women need to be told that the risks on the offspring are not known as it is a relatively new technique and therefore would be known in future. It needs to be mentioned that very few women return for using the stored oocytes and therefore the debate is ongoing whether it is worth the risks involved in the procedure.

\section{Ovarian Tissue Cryopreservation}

Ovarian cortex freezing and autotransplantation were performed initially by Donnez et al. and the first human baby was born in $2004 .^{9}$ Thereafter, many successful pregnancies have been reported worldwide. In prepubertal girls diagnosed with malignancy where the chemotherapeutic agent would lead to fertility loss, ovarian tissue cryopreservation can be considered. This has been used in women with breast cancer and hematological malignancies. Lot of experience has been gained in ovarian tissue freezing and subsequent reimplantation. Both procedures are performed by laparoscopy, and therefore it involves multidisciplinary approach. The procedure involves removing a part of the ovary and extracting the ovary and transporting to a place where it is processed and frozen as small strips of cortex. The medulla that contains small immature oocytes is also processed and immature oocytes are also frozen. ${ }^{10}$ This process as a whole involves counseling, consent, and assessment of fitness to undergo such a procedure. In case of prepubertal girls, consent of parents or legal guardians is taken. The indications of ovarian cortex freezing have also included benign diseases like SLE, women at risk of POI due to need for alkylating chemotherapy for autoimmune diseases, and also in endometriosis.

Once the disease is considered treated, the ovarian cortex is thawed and transplanted in the pelvis laparoscopically, on the ovary/peritoneal surface, and sutured or a pocket is made by opening the peritoneum and covered. This kind of reimplantation is called orthotopic transplantation. When the ovarian tissue is reimplanted below the skin away from origin, then it is called heterotopic transplantation. This is not preferred because the skin temperature decreases the graft age and more failures are recorded even though the oocyte retrieval is easier.

The ovarian tissue transplantation has also been attempted between twin sisters, in women with POI with successful pregnancy. There are few publications on this as ovum donation from a younger fertile woman is considered a less invasive option with more success. ${ }^{9}$ Ovarian transplantation (whole) has also been attempted but due to small vessels supplying the ovary it has not proven to be of success.

These procedures have ethical and safety concerns as they are performed in prepubertal girls and women suffering from life-threatening or debilitating conditions where in some cases the survival is questionable, therefore it poses a dilemma both for physician, the surgeon who has to perform this in high-risk conditions, and for the woman or her parents who have psychological burden of decision making. Absolute safety cannot be guaranteed as there are reports of deaths during surgery and in the postoperative period, when ovarian tissue freezing and reimplantation were attempted in malignant as well as benign conditions. ${ }^{10,11}$

Recently, ovarian tissue freezing and autotransplantation have been reported in women desiring to delay menopause or in women who wish to avoid aging due to hormonal withdrawal. Small ovarian cortex slices are transplanted at the heterotopic site, which had been cryopreserved at a younger age. This avoids the need of hormone replacement therapy in these aging women. Here too lies the ethical dilemma whether a surgical procedure should be offered at a young age in anticipation of aging.

\section{In Vitro Maturation}

This is at the experimental stage where the removed ovarian cortex is processed in vitro and cultured so as to retrieve few oocytes from this tissue, which are further cultured in vitro to the optimal size and then as a third step fertilized. ${ }^{12}$ This has been successful in animals but study of human oocytes awaits results. The other application of in vitro maturation is when the unstimulated ovary is aspirated and the immature oocytes retrieved are cultured in vitro, which are fertilized and frozen as embryos or the in vitro matured oocytes are cryofreezed for use in future. This is not easy and there is limited success due to difficulty in aspiration of the small unstimulated ovary and fewer number of oocytes are retrieved. The attempt to freeze the immature oocytes is of limited success but may be possible in future. The immature oocytes are also retrieved from the ovarian tissue during preparation of the ovarian cortex for freezing.

\section{Ovarian Protection}

Ovarian protection techniques are surgical and nonsurgical where these methods are used to prevent and protect the ovaries from the gonadotoxic irradiation or chemotherapy. This is an effort to decrease the dose or impact of the therapy where other techniques of fertility preservation cannot be advocated and therapy needs to instituted.

The evidence presently does not support use of the gonadotropinreleasing hormone $(\mathrm{GnRH})$ analog, but this is the safest method when other methods cannot be advocated. Administration of $\mathrm{GnRH}$ analogs 
before and during chemotherapy suppresses the ovary causing decreased number of growing primordial follicles entering the pool, therefore making them less sensitive to gonadotoxic chemotherapeutic agents. Gonadotropin-releasing hormone suppresses the pituitary function and this indirectly suppresses the ovary. There may be a direct protective effect of $G n R h$ analogs on the ovarian germline stem cells. ${ }^{13}$

\section{Ovarian Transposition}

In women with carcinoma of cervix where the ovaries are spared, considering a probable need for pelvic irradiation, the ovaries are transpositioned at a higher site and fixed to the peritoneum by sutures either during laparoscopy or laparotomy moving them away from the field of irradiation. ${ }^{12}$ This procedure is called as ovarian transposition or oophoropexy. As per American Society of Clinical Oncology guidelines, use of transposition of ovaries in preserving fertility is debatable and variable according to the irradiation dosage, the site, kind of pelvic irradiation, age, and also whether chemotherapy is required. ${ }^{12}$

\section{Conclusion}

Fertility preservation is being discussed and practiced worldwide and now most oncological centers globally have oncofertility clinics to discuss, counsel women about the impact of the therapy, and give them the options to choose and right to decide for themselves. More awareness is needed among physicians to protect themselves by appropriate consent and counseling as the overall survival in childhood malignancies and cancers diagnosed in reproductive years has improved.

\section{References}

1. Woodruff TK. The oncofertility consortium - addressing fertility in young people with cancer. Nat Rev Clin Oncol 2010;7(8):466-475. DOI: $10.1038 /$ nrclinonc.2010.81.
2. Wallace WH, Smith AG, Kelsey TW, et al. Fertility preservation for girls and young women with cancer: population-based validation of criteria for ovarian tissue cryopreservation. Lancet Oncol 2014;15(10):1129-1136. DOI: 10.1016/S1470-2045(14)70334-1.

3. Gidoni $\mathrm{Y}$, Holzer $\mathrm{H}$, Tulandi T, et al. Fertility preservation in patients with non-oncological conditions. Reprod Biomed Online 2008;16(6):792-800. DOI: 10.1016/S1472-6483(10)60144-7.

4. Goldman KN. Elective oocyte cryopreservation: an ounce of prevention? Fertil Steril 2018;109(6):1014-1015. DOI: 10.1016/ j.fertnstert.2018.03.023.

5. Ethics Committee of the American Society for Reproductive Medicine. Planned oocyte cryopreservation for women seeking to preserve future reproductive potential: an ethics committee opinion. Fertil Steril 2018;110(6):1022-1028. DOI: 10.1016/j.fertnstert.2018.08.027.

6. Bedoschi G, Oktay K. Current approach to fertility preservation by embryo cryopreservation. Fertil Steril 2013;99(6):1496-1502. DOI: 10.1016/j.fertnstert.2013.03.020.

7. Tozzo P, Fassina A, Nespeca P, et al. Understanding social oocyte freezing in Italy: a scoping survey on university female students' awareness and attitudes. Life Sciences, Soc Policy 2019;15(1):2-14.

8. Jones BPI, Saso S, Mania, Smith JR, et al. The dawn of a new ice age: social egg freezing. Acta Obstetricia et Gynecologica Scandinavica 2018;97(6):641-647. DOI: 10.1111/aogs.13335.

9. Donnez J, Dolmans MM. Ovarian tissue freezing: current status. Curr Opin Obstet Gynecol 2015;27(3):222-230. DOI: 10.1097/ GCO.0000000000000171.

10. Practice Committee of the American Society for Reproductive Medicine. Ovarian tissue cryopreservation: a committee opinion. Fertil Steril 2014;101(5):1237-1243. DOI: 10.1016/j.fertnstert.2014.02.052.

11. Leonel ECR, Lucci CM, Amorim CA. Cryopreservation of human ovarian tissue: a review. Transfus Med Hemother 2019;46(3):173-181. DOI: 10.1159/000499054.

12. Salama M, Mallmann P. Emergency fertility preservation for female patients with cancer: clinical perspectives. Anticancer Res 2015;35(6):3117-3128.

13. Kumar P, Sharma A. Gonadotropin-releasing hormone analogs: understanding advantages and limitations. J Hum Reprod Sci 2014;7(3):170-174. DOI: 10.4103/0974-1208.142476. 